\title{
Error Reduction of Modified Booth Multipliers in Mac Unit
}

\author{
K.Santhana Lakshmi ${ }^{1}$, G. Brindha ${ }^{2}$, A.Andamuthu ${ }^{3}$ \\ Assistant Professor ${ }^{1}$, Assistant Professor ${ }^{2}$, Assistant Professor ${ }^{3}$ \\ Department of Electronics and Communication Engineering, Muthayammal Engineering College.
}

\begin{abstract}
The fixed-width multiplier is well attractive to many multimedia and digital signal processing systems. It proposes a reduction of truncation error from 16-bit to 8-bit MSB bits (Truncated output) using simple error reduction circuit. The Fixed width modified booth multiplier is used to minimize the partial product matrix of Booth multiplication. Multiplication is binary mathematical operation scaling one number by another. Lead the design of high accuracy, low power and area in MAC unit and compare with the Wallace tree multiplier. The system will be designed using VHDL coding (Very High speed Integrated Circuit Hardware Descriptive Language).
\end{abstract}

Index Terms: Multiplier and Accumulator, Most significant bits, Modified booth multiplier, error reduction circuit, fixed width multiplier.

\section{Introdution}

A multiplier is a factor of proportionality that measures how much an endogenous variable changes in response to a change in some exogenous variable. Multipliers are the basic and important building blocks of VLSI systems. The technology considers the power consumption, area and accuracy.

Accuracy is one of the backbones of the multipliers. To achieve the high performance of modified booth encoding which reduces the partial products. $\mathrm{N} * \mathrm{~N}$ fixed width multipliers that generate only the $\mathrm{N}$ most significant bits (MSB) and to maintain a fixed word size. Truncation error will be high in this type of multiplier.

This truncation error can be reduced by using error reduction circuit; it can be added estimated carry value of the reversed adder cells. For reducing the partial product output means, the truncation error will be occurring. The final output of partial product is used in MAC unit, DSP applications, and multimedia.

To get better error performance with a simple error reduction circuit, Booth encoded outputs are to generate the error reduction value.

By modified booth encoding, which groups the bits of the multiplier into triplets. Output of MBE (Modified Booth Encoder) is given to the input of Partial Product. Used to reduce the partial product steps, using truncation method to reduce the partial product from 16 bit to 8bit, that is truncating the LSB (Least Significant Bits) bits only.

\section{Modified Booth Encoder}

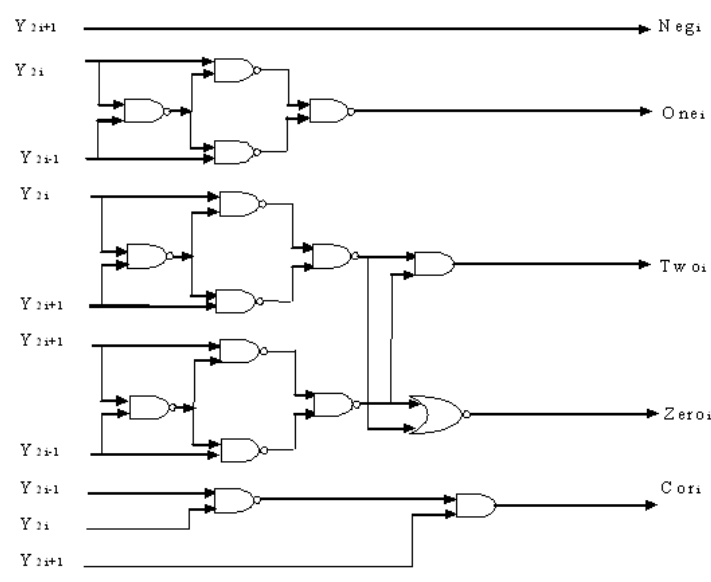

Fig.1. Circuit diagram of modified booth encoder

Conditions:

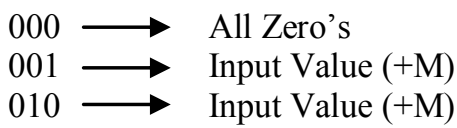




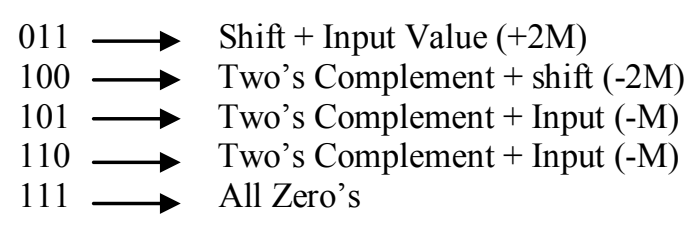

The above diagram has $\mathrm{Y}$ inputs and produces the output as Onei, Twoi, Zeroi, and Cori. Using $8 * 8$ means take only the output as 8 (Instead of 16) that are called fixed width. The output of modified booth encoder is given to the input of partial product.

101 and 110, the output becomes two's complement and input value. In partial product the output comes only the One's complement, need two's complement means adding carry value1, get two's complement output.

\begin{tabular}{|c|c|c|c|c|c|c|c|c|}
\hline$Y_{2 i+1}$ & $Y_{21}$ & $Y_{2 \mathrm{i}-1}$ & Function & N egi & Onei & T woi & Zeroi & Cori \\
\hline 0 & 0 & 0 & 0 & 0 & 1 & 0 & 0 & 0 \\
\hline 0 & 0 & 1 & $+\mathrm{M}$ & 0 & 0 & 1 & 0 & 0 \\
\hline 0 & 1 & 0 & $+\mathrm{M}$ & 0 & 0 & 1 & 0 & 0 \\
\hline 0 & 1 & 1 & $+2 \mathrm{M}$ & 0 & 0 & 0 & 1 & 0 \\
\hline 1 & 0 & 0 & $-2 \mathrm{M}$ & 1 & 0 & 0 & 1 & 1 \\
\hline 1 & 0 & 1 & $-M$ & 1 & 0 & 1 & 0 & 1 \\
\hline 1 & 1 & 0 & $-M$ & 1 & 0 & 1 & 0 & 1 \\
\hline 1 & 1 & 1 & 0 & 1 & 1 & 0 & 0 & 0 \\
\hline
\end{tabular}

A. Function of Modified Booth Multiplier

An example of Modified Booth Multiplier is in below:

$\mathrm{X} \rightarrow 11010010 \quad$ (Multiplicand)

$\mathrm{Y} \rightarrow 11010011 \quad$ (Multiplier)

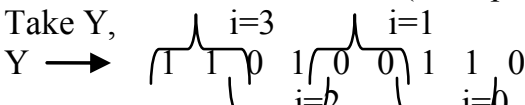

Condition: $i=0$ to $3 ; j=0$ to 7 ;

For $\mathrm{i}=0 \longrightarrow 110$

$\mathrm{Y} 2(0)+1=1 \rightarrow \mathrm{Neg}(0)=1$

$\mathrm{Y} 2(0)=1 ; \mathrm{Y} 2(0)-1=0 \rightarrow \mathrm{One}(0)=1$

$\mathrm{Y} 2(0)=1 ; \mathrm{Y} 2(0)+1=1 \rightarrow \mathrm{Two}(0)=0$

$\mathrm{Y} 2(0)+1=1 ; \mathrm{Y} 2(0)-1=0 \longrightarrow \operatorname{Zero}(0)=0$

$\mathrm{Y} 2(0)-1=0 ; \mathrm{Y} 2(0)=1 ; \mathrm{Y} 2(0)+1=1 \longrightarrow \operatorname{Cor}(0)=1$

For $\mathrm{i}=1 \longrightarrow 001$

$\mathrm{Y} 2(1)+1=1 \rightarrow \mathrm{Neg}(1)=0$

$\mathrm{Y} 2(1)=1 ; \mathrm{Y} 2(1)-1=0 \longrightarrow$ One(1)=1

$\mathrm{Y} 2(1)=1 ; \mathrm{Y} 2(1)+1=1 \rightarrow \mathrm{Two}(1)=0$

$\mathrm{Y} 2(1)+1=1 ; \mathrm{Y} 2(1)-1=0 \rightarrow \operatorname{Zero}(1)=0$

$\mathrm{Y} 2(1)-1=0 ; \mathrm{Y} 2(1)=1 ; \mathrm{Y} 2(0)+1=1 \longrightarrow \operatorname{Cor}(1)=0$

For $\mathrm{i}=\mathrm{Z} \longrightarrow 010 \quad \mathrm{Y} 2(2)+1=1 \quad \mathrm{Neg}(0)=0$

$\mathrm{Y} 2(2)=1 ; \mathrm{Y} 2(2)-1=0 \rightarrow \mathrm{One}(2)=0$

$\mathrm{Y} 2(2)=1 ; \mathrm{Y} 2(2)+1=1 \rightarrow \operatorname{Two}(2)=1$

$\mathrm{Y} 2(2)+1=1 ; \mathrm{Y} 2(2)-1=0 \rightarrow \mathrm{Zero}(2)=0$

$\mathrm{Y} 2(2)-1=0 ; \mathrm{Y} 2(2)=1 ; \mathrm{Y} 2(2)+1=1 \longrightarrow \operatorname{Cor}(2)=0$

For $\mathrm{i}=3 \longrightarrow 110$

$\mathrm{Y} 2(3)+1=1 \rightarrow \operatorname{Neg}(0)=1$

$\mathrm{Y} 2(3)=1 ; \mathrm{Y} 2(3)-1=0 \longrightarrow \operatorname{One}(3)=1$

$\mathrm{Y} 2(3)=1 ; \mathrm{Y} 2(3)+1=1 \rightarrow \mathrm{Two}(3)=0$

$\mathrm{Y} 2(3)+1=1 ; \mathrm{Y} 2(3)-1=0 \rightarrow \mathrm{Zero}(3)=0$

$\mathrm{Y} 2(3)-1=0 ; \mathrm{Y} 2(3)=1 ; \mathrm{Y} 2(3)+1=1 \longrightarrow \operatorname{Cor}(3)=1$

\section{Partial Product}

The values of partial product bits are heavily dependent on the outputs of booth encoder. It explores the relation between the outputs of booth encoders and the carry value propagated from LPminor to LPmajor. 


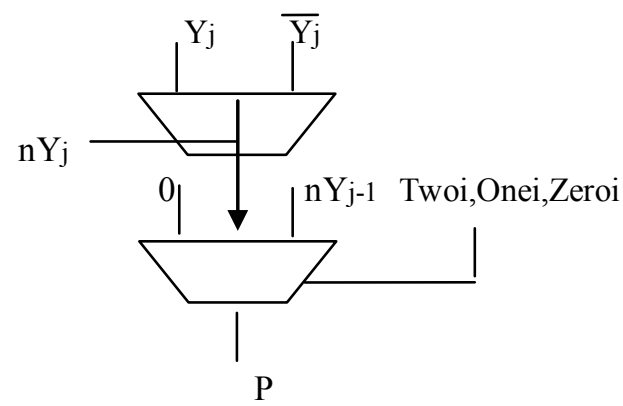

Fig.2. Circuit diagram of Partial Product

Multiplexer have $\mathrm{Y}$ inputs, $\mathrm{P}$ output, selection inputs ( 0 or 1$) \mathrm{P}$ will be equal to one of the inputs, depending upon the selection inputs. Minimum number of sign extension. The output is checked by using VHDL programming technique.

A. Function of Partial product

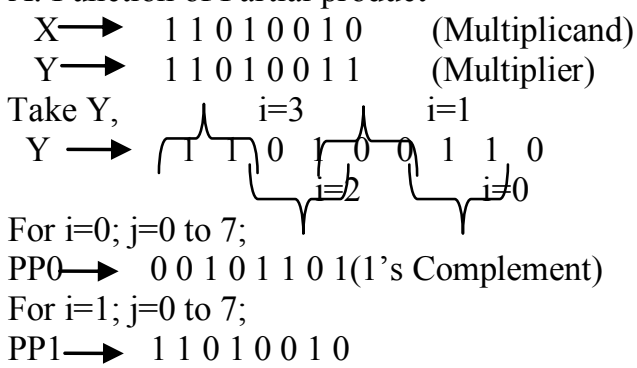

For $\mathrm{i}=2 ; \mathrm{j}=0$ to 7 ;

$\mathrm{PP} 2 \longrightarrow 11010010$

For $\mathrm{i}=3 ; \mathrm{j}=0$ to 7 ;

PP3 $\rightarrow 00101101$ (1's Complement)

For $\mathrm{i}=0$

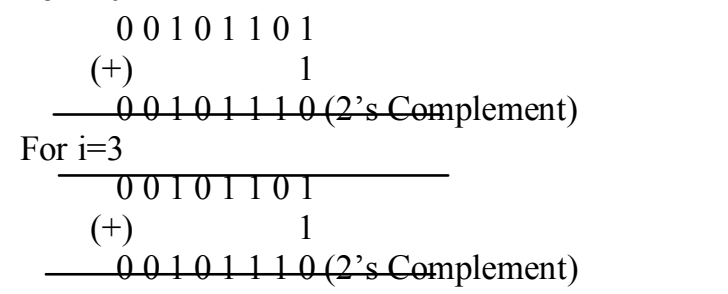

In the above calculation, only getting the ones complement output. 110 is the two's complement type, but the partial product output is ones complement, so need to add correction bit 1.Finally getting the twos complement output.

B. Partial Product Outputs

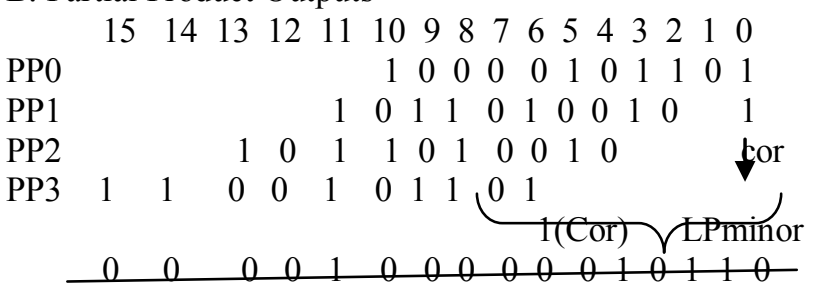

Fig.3. Calculation of Partial Product

To truncate the LPminor parts , adding carry value 1 or 0 in LP(Least Product)major parts, if carry is 0 means the output of 100 (PP0 of 10,9,8th digit)is same or carry is 1 means , the output of 100 becomes 101 .

In above figure, LSB (Lest Significant Bits) bits are split into LP(Lest Product) major and LP(Least Product) minor parts. LP minor star9ts from digit 0 to 6 and LP minor parts starts $7^{\text {th }}$ digit only. From $8^{\text {th }}$ to $15^{\text {th }}$ bit is the MSB(Most Significant Bits) bits

Adding carry value in below diagram,

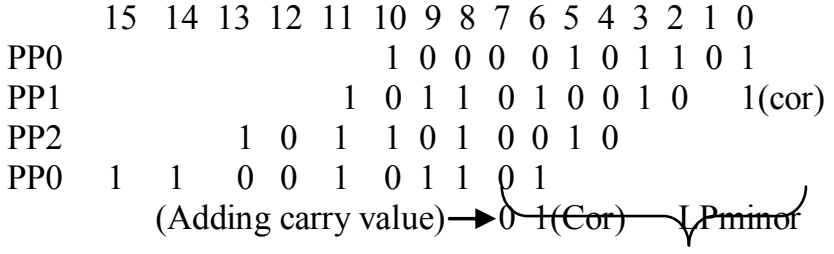


$\begin{array}{llllllllll}1 & 1 & 1 & 1 & 1 & 0 & 1 & 1 & 1 \\ \end{array}$

Fig.4. Adding Carry value in Partial Product

In fig4, the output is same there is no error output so move to next example, to reducing the bits from 16 to 8.The example becomes,

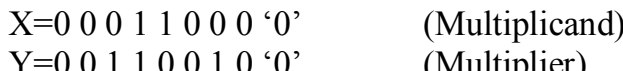

$$
\begin{aligned}
& \mathrm{Y}=001110010^{\circ} 0 \text { ' (Multiplier) } \\
& 100 \longrightarrow \mathrm{i}=0 \\
& 001 \longrightarrow \mathrm{i}=1 \\
& 110 \longrightarrow \mathrm{i}=2 \\
& \mathrm{O} 01 \longrightarrow \mathrm{i}=3
\end{aligned}
$$

The Output becomes,

$100=11001111$

$001=000011000$

$110=11100111$

$001=11001111$

100 becomes the two's complement output. There is no change in adding carry value in few types of partial product inputs. In above calculations have some changes in adding carry value of partial product that is trucation error wiil occur.

To overcome the truncation, using simple error reduction circuit, truncation means eliminating the least significant bits.

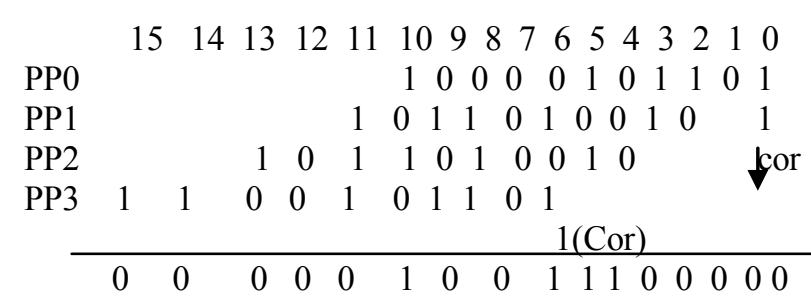

Fig.5. Example of Partial Product

Adding carry value in below diagram is,

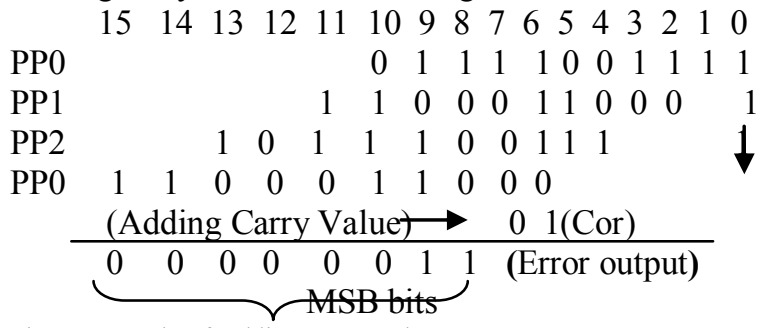

Fig.6. Example of Adding carry Value

\section{Truncation Error}

Truncation is the term for limiting the number of digits, discarding the LSB bits. It occurs, when a number cannot be fully represented due to memory limitations. Truncating would yield the same result as rounding, but truncation does not round up or round down the digits; it merely cuts off at the specified digit.

Multiplication is required in Digital signal processing. A substantial hardware savings is realized by summing only the $\mathrm{n}+\mathrm{k}$ most significant columns of the matrix. This method of multiplication is called truncated multiplication. Truncated multiplication leads to two sources of error: reduction error and rounding error. Reduction error occurs because the $n-k$ least significant columns of the multiplication matrix is not used to compute the product. Rounding error occurs because the product is rounded to $\mathrm{n}$ bits.

\section{Summary And Results}

The output of modified booth encoder is given to the input of partial product for reducing the partial product steps and to produce the uniformity of the modified booth encoder. Using truncation method (Adding carry value), for reducing from 16 bit output to 8 bit output. In the future, I have to design an error reduction circuit and apply the MAC (Multiplier and Accumulator) unit and finally compare the Wallace tree Multiplier.
A. Modified booth Encoder Output 
The modified booth encoder has two inputs $\mathrm{X}$ and $\mathrm{Y}$. Only take $\mathrm{Y}$ as input and the output becomes Negation,Zero,One,Correction,Two.

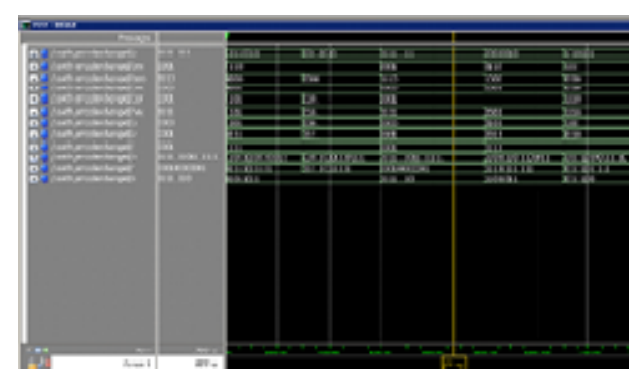

By modified booth encoding, which groups the bits of the multiplier into triplets. The output of Modified booth encoder is given to the input of Partial Product.

\section{B. product, sum and carry of PP Output}

The inputs are $\mathrm{X}$ and $\mathrm{Y}$. Take $\mathrm{Y}$ as an input, these inputs are splitting into PP0, PP1, PP2, PP3.Depending on the input value the output becomes changed. T he output has Sum, Carry and Product.

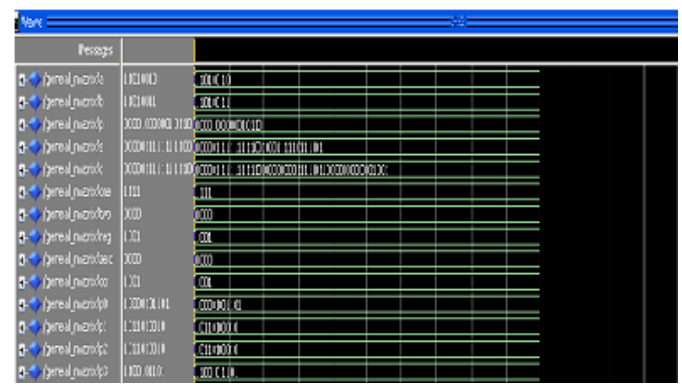

\section{Adding carry value Output} adder, full adder.

The splitting of the (Partial Product) PP0, PP1, PP2, PP3 and adding this partial products using half

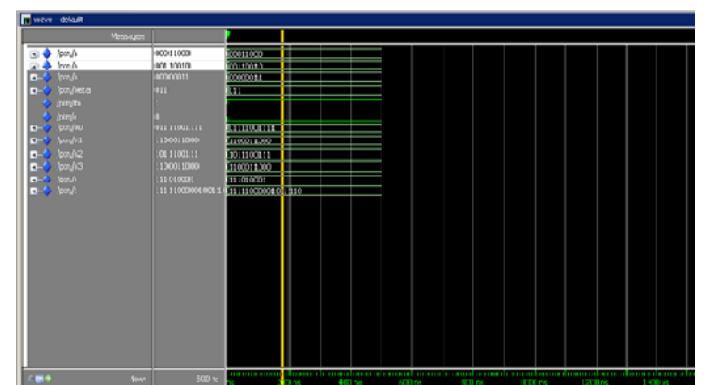

The reduction of 16 bit into 8 bit by using LSB bits and MSB bits .the LSB bits are split into LP major and LP minor parts. To eliminate the LP minor parts means some error occur in output, so adding carry value 1 or 0 based on input values.

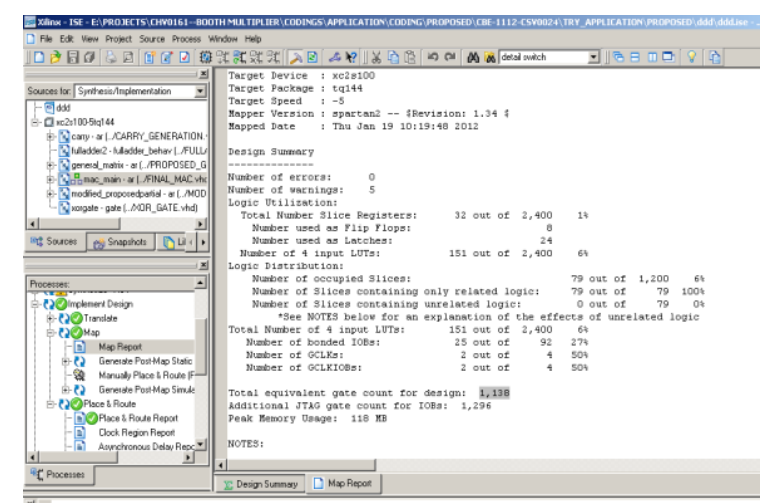

Fig.7 Area for modified booth encoder 

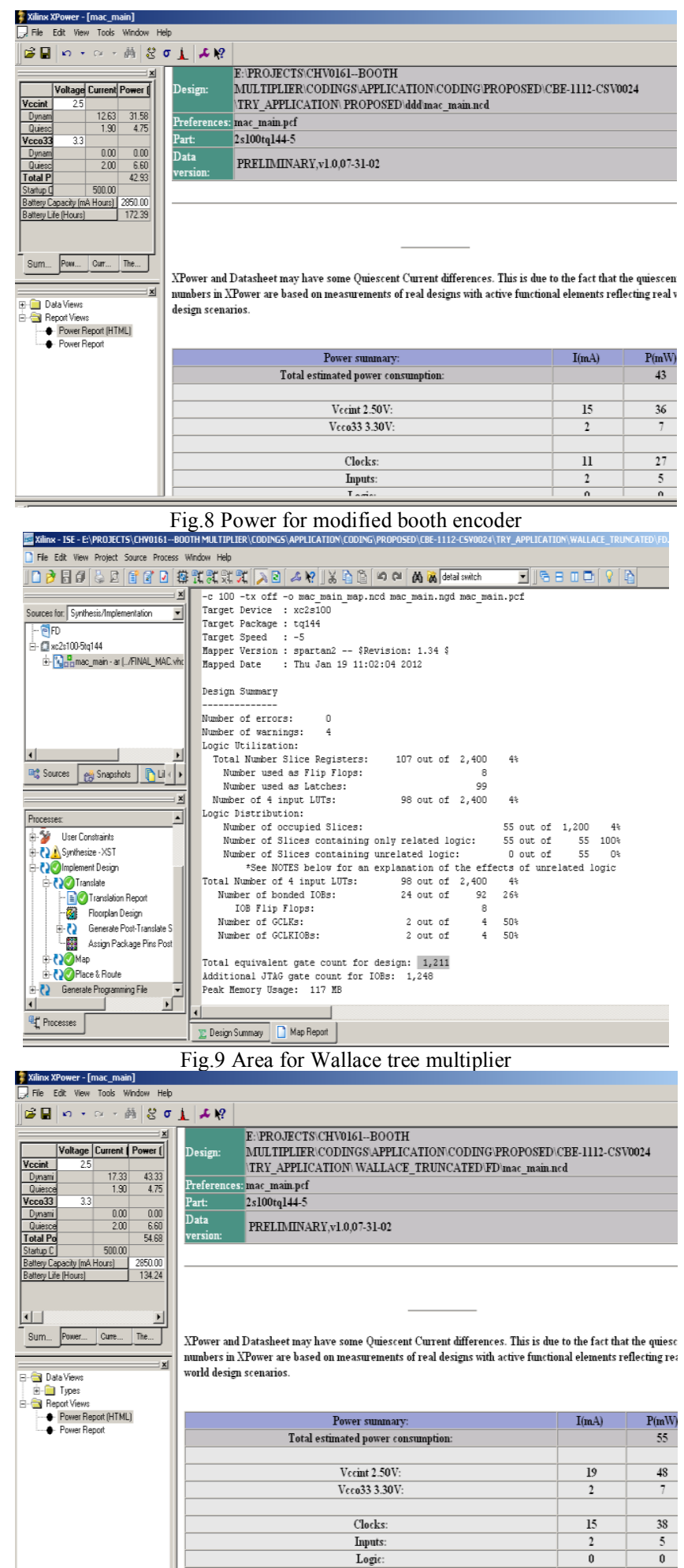

Fig.10 Power for Wallace tree multiplier

Table.1 Comparison of Wallace tree multiplier and Modified booth encoder with MAC unit

\begin{tabular}{|c|c|c|}
\hline Parameters & $\begin{array}{c}\text { Modified booth } \\
\text { multiplier }\end{array}$ & $\begin{array}{c}\text { Wallace tree } \\
\text { multiplier }\end{array}$ \\
\hline Power Consumption & $43(\mathrm{mw})$ & $55(\mathrm{mw})$ \\
\hline Gate Counts & 1,138 & 1,211 \\
\hline Number of slices & 79 out of 1,200 & 55 out of 1,200 \\
\hline Number of 4input LUT's & 151 out of 2,400 & 98 out of 2,400 \\
\hline $\begin{array}{c}\text { Number of bonded } \\
\text { DOB's }\end{array}$ & 25 out of 92 & 24 out of 92 \\
\hline
\end{tabular}




\section{References}

[1] Jiun-Pi Wang,Shiann-Rong Kuang ,Shish-Chiang Liang IEEE Trans on VLSI "High accuracy fixed width modified booth multipliers",vol 19,No.1,PP.52-60,January 2011.

[2] Kyung-Ju Cho, Kwang-Chul Lee, Jin-Gyun Chung, Member, IEEE, and Keshab K. Parhi, Fellow, IEEE, ” Design of Low-Error FixedWidth Modified Booth Multiplier", IEEE Transactions on Very Large scale Integration (VLSI) Systems, Vol. 12, No. 5,PP.522-531, May 2004.

[3] Wen-Chang Yeh and Chein-Wei Jen, "High-Speed Booth Encoded Parallel Multiplier Design”, IEEE Transactions on Computers, Vol. 49, No. 7, PP.692-700, July 2000.

[4] L. D. Van and C. C. Yang, "Generalized low-error area-efficient fixed width multiplier," IEEE Trans. Circuits Syst. I, Reg. Papers, vol. 52, No.8, PP.1608-1619, August. 2005.

[5] F.Elguibaly, "A fastparallel multiplier-accumulator using the modified Booth algorithm,” IEEE Trans. Circuits Syst. II, vol. 47, No. 9, PP.902-908, September 2000.

[6] Oscal T.-C. Chen, Sandy Wang, and Yi-Wen Wu," Minimization of Switching Activities of Partial Products for Designing Low-Power Multipliers", IEEE Transactions on very Large Scale Integration (VLSI) Systems, Vol. 11, No. 3,PP.418-432, June 2003.

[7] C. N.Marimuthu, P. Thangaraj, "Low Power High Performance Multiplier", ICGST-PDCS, Vol.2, No.3, PP.12-22, December 2008.

[8] J. M. Jou, S. R. Kuang, and R. D. Chen, "Design of low-error fixed widthmultiplier for DSP applications,” IEEE Trans. Circuits Syst. I,Exp. Briefs, vol. 46, no. 6, PP. 836-842, June 1999 